\title{
Validation of the Lewis score for the evaluation of small-bowel Crohn's disease activity
}

Authors

Institutions
José Cotter ${ }^{1,3}$, Francisca Dias de Castro ${ }^{1}$, Joana Magalhães ${ }^{1}$, Maria João Moreira ${ }^{1}$, Bruno Rosa ${ }^{1}$

Gastroenterology Department, Centro Hospitalar do Alto Ave, Guimarães, Portugal

${ }^{2}$ Life and Health Sciences Research Institute, School of Health Sciences, University of Minho, Braga, Portugal

${ }^{3}$ Life and Health Sciences Research Institute/3B's, PT Government Associate Laboratory, Braga/Guimarães, Portugal submitted 15. June 2014 accepted after revision: 22. September 2014

\section{Bibliography}

Dol http://dx.doi.org/ 10.1055/s-0034-1390894 Published online: 2014 Endoscopy (c) Georg Thieme Verlag KG Stuttgart · New York ISSN 0013-726X

Corresponding author José Cotter, MD

Gastroenterology Department Centro Hospitalar do Alto Ave Rua dos Cutileiros Creixomil

Guimarães 4835-044

Portugal

Fax: +351-253-421308

jcotter@chaa.min-saude.pt
Background and study aims: The Lewis score was developed to measure mucosal inflammatory activity as detected by small-bowel capsule endoscopy (SBCE). The aim of the current study was to validate the Lewis score by assessing interobserver correlation and level of agreement in a clinical setting.

Patients and methods: This was a retrospective, single-center, double-blind study including patients with isolated small-bowel Crohn's disease who underwent SBCE. The Lewis score was calculated using a software application, based on the characteristics of villous edema, ulcers, and stenoses. The Lewis score was independently calculated by one of three investigators and by a central reader (gold standard). Interobserver agreement was assessed using intraclass correlation (ICC) coefficient and Bland-Altman plots.

Results: A total of 70 patients were consecutively included (mean age $33.9 \pm 11.7$ years). The mean Lewis score was 1265 and 1320 for investigators and the central reader, respectively. There was a

\section{Introduction}

$\nabla$

The role of small-bowel capsule endoscopy (SBCE) in the evaluation of patients with known or suspected Crohn's disease is still evolving. The small bowel is involved in approximately $80 \%$ of patients with Crohn's disease, and in up to one-third of cases the small bowel is the only segment of the digestive tract affected by the disease [1]. Moreover, SBCE has consistently demonstrated the highest diagnostic yield when compared with other small-bowel imaging modalities for the detection of mucosal lesions suggestive of Crohn's disease, particularly for mild lesions and those located in the proximal small bowel [2,3]. SBCE may be useful to confirm or exclude the diagnosis in patients with suspected Crohn's disease, and it may also be used to assess disease extent and activity in patients with a previously established di- high correlation, both for scores obtained for each tertile (first tertile $r=0.659-0.950$, second tertile $r=0.756-0.906$, third tertile $r=0.750$ $0.939)$, and for the global score $(r=0.745-0.928)$ $(P<0.0001)$. Interobserver agreement was almost perfect between the investigators and the central reader (first tertile ICC $=0.788-0.971$, second tertile ICC $=0.824-0.943$, third tertile ICC $=0.857$ 0.968 , global score ICC $=0.852-0.960 ; P<0.0001$ ). The inflammatory activity was classified as normal (score $<135)$ in $2.9 \%$ vs. $2.9 \%$, mild (score $\geq$ $135-<790)$ in $51.4 \%$ vs. $55.7 \%$, and moderate to severe (score $\geq 790$ ) in $45.8 \%$ vs. $41.4 \%$ of patients, respectively $(P<0.001)$.

Conclusion: A strong interobserver agreement was demonstrated for the determination of the Lewis score in a practical clinical setting, validating this score for the reporting of small-bowel inflammatory activity. The Lewis score might be used for diagnosing, staging, follow-up, and therapeutic assessment of patients with isolated small-bowel Crohn's disease.

agnosis of Crohn's disease. However, endoscopic findings are nonspecific and must be carefully interpreted within the proper clinical context [4]. The availability of an objective and reproducible measuring instrument, with good interobserver agreement, seems essential for the assessment of inflammatory activity and extent. Such an instrument would also help to standardize the terminology and method of interpretation of lesions that are consistent with Crohn's disease, and establish cutoff levels indicative of the severity of inflammation. The classical threshold of more than three ulcers to diagnose Crohn's disease as proposed by Mow et al. [5] has been widely used, although it does not evaluate the size or distribution of the ulcers or account for other lesions typical of Crohn's disease, such as villous edema or stenoses. Moreover, it does not define cutoff levels to classify inflammatory activity according to 
grade of severity, and it has been shown to yield a positive predictive value for the diagnosis of Crohn's disease of only $50 \%-69 \%$ [6].

The recently introduced quantitative scoring systems such as the Lewis score [7] and the capsule endoscopy Crohn's disease activity index (CECDAI or Niv Score) [8] have overcome some of these limitations. The CECDAI has been validated recently in a multicenter prospective study [9], whereas the Lewis score has not yet undergone such validation. Nevertheless, the Lewis score, a cumulative scoring system based on the characteristics and distribution of villous edema, ulceration, and stenosis, has been converted into a user-friendly software application, which calculates the score automatically. With this application, the small bowel is automatically divided into equal thirds (tertiles) based on the transit time of the capsule. For each tertile, a numeric subscore (based on the extent and distribution of edema, and the number, size, and distribution of ulcers) is calculated. The final score is calculated by adding the score for the worst-affected tertile to the score for stenosis (single/multiple, ulcerated/not ulcerated, traversed/not traversed by the capsule):

Lewis score $=$

inflammatory score of the worst-affected tertile [(villous parameter $\times$ extent $\times$ descriptor $)+($ ulcer number $\times$ extent $\times$ size $)]+$ stenosis score (number $\times$ ulcerated $\times$ traversed).

The Lewis score classifies small-bowel inflammatory activity into three grades of severity of inflammation: 1) normal or clinically insignificant mucosal inflammatory change (score <135); 2) mild disease (score $\geq 135-<790$ ); and 3) moderate-to-severe disease (score $\geq 790$ ). Although widely used, this score has not yet been externally validated for use in clinical practice.

The aim of this study was to assess the interobserver agreement of the Lewis score, and to validate the use of this scoring system in clinical practice for the assessment of small-bowel Crohn's disease.

\section{Patients and methods}

$\nabla$

A retrospective, single-center, double-blind study was conducted between May 2008 and August 2013, and included all consecutive patients with isolated nonstricturing and nonpenetrating small-bowel Crohn's disease who underwent SBCE. Inclusion criteria were age between 17 and 75 years, and known Crohn's disease of the small bowel. In the absence of a single gold standard to diagnose Crohn's disease, patients eligible to enter the study had an established diagnosis of Crohn's disease based on follow-up data with all available clinical, analytical, imaging, endoscopic, and histological elements. Patients covering the full spectrum of disease activity, from remission to severe disease, were included in the study. Patients with obstructive symptoms and/or those with evidence of ileal stenosis at ileocolonoscopy and/or radiological features of stricturing or penetrating disease were not eligible for SBCE and were thus excluded from the study. Patients taking aspirin or nonsteroidal anti-inflammatory drugs (NSAIDs) were instructed to discontinue the medication at least 4 weeks before the SBCE examination. Patients followed a clear liquid diet for 24 hours and fasting for 12 hours prior to SBCE (PillCam SB2; Given Imaging Ltd., Yoqneam, Israel).

Following the examination, the SBCE videos were reviewed and the Lewis score was calculated by one of three investigators (investigator 1 [F.D.C.], investigator 2 [J.M.], investigator 3 [M.J.M.]), using the software application in the RAPID Reader v.6 or v.7 workstation (Given Imaging). In addition, all examinations were independently reviewed by one experienced reader (central reader [B.R.]), who was blinded to the results of the other investigators. The report from the central reader represented the endoscopic gold standard.

Using the software application to calculate the Lewis score, the small bowel was automatically divided into equal thirds (tertiles) based on the transit time of the capsule. In those cases where the capsule did not reach the cecum, small-bowel tertiles were determined based on the last small-bowel image available and the relative position of the capsule to the ileocecal valve, as estimated using topographic landmarks with the localization track of the RAPID software. For each tertile, a numeric subscore (based on the extent and distribution of edema, and the number, size, and distribution of ulcers) was calculated. The final score was calculated as the cumulative sum of the worst-affected tertile considering villous edema and ulcers, plus the score for stenosis (single/ multiple, ulcerated/not ulcerated, traversed/not traversed by the capsule); the stenosis score was determined for the whole examination only and not for each tertile ( $\bullet$ Table 1$)$. Small-bowel inflammatory activity was classified into three grades: 1) normal or clinically insignificant mucosal inflammatory change (score < 135); 2) mild disease (score $\geq 135-<790$ ); and 3) moderate-tosevere disease (score $\geq 790$ ).

\begin{tabular}{|c|c|c|c|}
\hline Parameters & Number & Longitudinal extent $^{1}$ & Descriptors \\
\hline \multirow{3}{*}{$\begin{array}{l}\text { Villous appearance } \\
\text { (worst-affected tertile) }\end{array}$} & Normal-0 & Short segment -8 & Single-1 \\
\hline & Edematous - 1 & Long segment- 12 & Patchy - 14 \\
\hline & & Whole tertile - 20 & Diffuse -17 \\
\hline \multirow{4}{*}{$\begin{array}{l}\text { Ulcer } \\
\text { (worst-affected tertile) }\end{array}$} & None $-0^{2}$ & Short segment -5 & $<1 / 4-9^{3}$ \\
\hline & Single $-3^{2}$ & Long segment- 10 & $1 / 4-1 / 2-12^{3}$ \\
\hline & Few $-5^{2}$ & Whole tertile - 15 & $>1 / 2-18^{3}$ \\
\hline & Multiple $-10^{2}$ & & \\
\hline \multirow{3}{*}{$\begin{array}{l}\text { Stenosis } \\
\text { (whole study) }\end{array}$} & None - 0 & Ulcerated-24 & Traversed - 7 \\
\hline & Single -14 & Nonulcerated - 2 & Not traversed -10 \\
\hline & Multiple-20 & & \\
\hline
\end{tabular}

Table 1 Lewis score (adapted from Gralnek et al. [7]).

Lewis score: Score of the worst-affected tertile [(villous parameter $\times$ extent $\times$ descriptor $)+($ ulcer number $\times$ extent $\times$ size $)]+$ stenosis score (number $\times$ ulcerated $\times$ traversed).

${ }^{1}$ Longitudinal extent: short segment: $<10 \%$ of the tertile; long segment: $11 \%-50 \%$ of the tertile; whole tertile: $>50 \%$ of the tertile.

2 Ulcer number: single: 1 ; few: $2-7$; multiple: $\geq 8$.

${ }^{3}$ Ulcer descriptor (size): proportion of the capsule picture filled by the largest ulcer. 


\section{Statistical analysis}

The mean Lewis score from each investigator was compared with that from the central reader using the paired samples $t$ test. The interobserver correlation, comparing the scoring results between the investigators and the central reader, was measured using the Pearson correlation coefficient, for both the individual quantitative scores for each tertile as well as the global small-bowel score. Pearson correlation coefficients were considered to be low if $r \leq 0.35$, moderate if $0.36<r<0.67$, high if $0.68<r<0.89$, and very high if $r>0.90$. Interobserver agreements between each investigator and the central reader were determined, both for each tertile and for the whole examination, using the intraclass correlation coefficient (ICC), and Bland-Altman plots were drawn for the global score. ICC was interpreted as follows: $0-$ 0.2 poor agreement; $0.3-0.4$ fair agreement; $0.5-0.6$ moderate agreement; $0.7-0.8$ strong agreement; and $>0.8$ almost perfect agreement. Statistical analysis was performed using SPSS (version 21.0; IBM Corp., Armonk, New York, USA) and MedCalc (version 14.8.1; MedCalc Software bvba, Ostend, Belgium). $P$ values of $<0.05$ were considered to be statistically significant.

\section{Results}

A total of 70 consecutive patients meeting the inclusion criteria were enrolled (mean age $33.9 \pm 11.7$ years [range 17-75]; 43 females [61.4\%]). The procedure was technically successful in all patients, and the videos were available for interpretation.

The mean Lewis score was $1265 \pm 1413$ (range 8-6040) and $1320 \pm 1602(112-6204)$ according to the investigators and the central reader, respectively. The comparison of mean Lewis score in each tertile and global examination between each investigator and the central reader is summarized in 0 Table 2 . Overall, there was a high or very high correlation between the investigators and the central reader both for scores obtained for each tertile $(r=0.659-0.950$ for the first tertile, $r=0.756-0.906$ for the second tertile, and $r=0.750-0.939$ for the third tertile), as well as for the global score $(r=0.745-0.928)(P<0.0001)$ $(\bullet$ Table 3). Interobserver agreement was almost perfect between the investigators and the central reader $($ ICC $=0.788$ 0.971 for the first tertile, ICC $=0.824-0.943$ for the second tertile, ICC $=0.857-0.968$ for the third tertile, and ICC $=0.852$ 0.960 for the global Lewis score $(P<0.0001)(\diamond$ Table 4; $\odot$ Fig. 1 , - Fig.2, and $\triangle$ Fig. 3).

Overall, small-bowel inflammatory activity was classified as normal (score $<135)$ in $2.9 \%$ vs. $2.9 \%$, mild (score $\geq 135-<790$ ) in $51.4 \%$ vs. $55.7 \%$, and moderate to severe (score $\geq 790$ ) in $45.8 \%$ vs. $41.4 \%$ of patients, respectively $(\mathrm{K}=0.7 ; P<0.001)$. The cecum was reached in $84.3 \%$ and $87.1 \%$ of examinations according to investigators and the central reader, respectively $(\mathrm{K}=0.884)$. Incomplete small-bowel examination was caused by delayed passage of the capsule at a previously unknown small-bowel stenosis in a minority of these patients $(n=4)$. These capsules were spontaneously excreted, without the need for further intervention. In the remaining examinations, the procedure was incomplete because of battery exhaustion before the capsule entered the cecum, as a result of delayed gastric passage or slow smallbowel transit. Overall, the diagnosis of small-bowel stenosis was established in $14.3 \%(n=10)$ and $18.6 \%(n=13)$ of patients, as evaluated by the investigators and the central reader, respectively $(\mathrm{K}=0.855)$.
Table 2 Comparison of mean Lewis score in each tertile and global examination between each investigator and the central reader: paired samples test.

\begin{tabular}{|c|c|c|c|}
\hline & Investigator & Central reader & $P$ \\
\hline \multicolumn{4}{|c|}{ Investigator 1 (F.D.C.) 24 examinations } \\
\hline First tertile & $241 \pm 307$ & $139 \pm 261$ & 0.047 \\
\hline Second tertile & $378 \pm 446$ & $218 \pm 333$ & 0.005 \\
\hline Third tertile & $833 \pm 700$ & $660 \pm 702$ & 0.100 \\
\hline Global score & $1464 \pm 1455$ & $1484 \pm 1744$ & 0.916 \\
\hline \multicolumn{4}{|c|}{ Investigator 2 (J.M.) 23 examinations } \\
\hline First tertile & $248 \pm 383$ & $187 \pm 229$ & 0.183 \\
\hline Second tertile & $431 \pm 797$ & $367 \pm 537$ & 0.561 \\
\hline Third tertile & $800 \pm 973$ & $686 \pm 724$ & 0.340 \\
\hline Global score & $1071 \pm 1441$ & $1235 \pm 1574$ & 0.475 \\
\hline \multicolumn{4}{|c|}{ Investigator $\mathbf{3}$ (M.J.M.) 23 examinations } \\
\hline First tertile & $342 \pm 480$ & $330 \pm 431$ & 0.702 \\
\hline Second tertile & $217 \pm 385$ & $221 \pm 323$ & 0.898 \\
\hline Third tertile & $699 \pm 749$ & $576 \pm 771$ & 0.037 \\
\hline Global score & $1234 \pm 1533$ & $1254 \pm 1377$ & 0.871 \\
\hline
\end{tabular}

Table 3 Correlations in each tertile and global examination between each investigator and the central reader: Pearson correlation coefficient.

\section{Correlation coefficient $\quad P$}

(r)

\begin{tabular}{|c|c|c|}
\hline \multicolumn{3}{|c|}{ Investigator 1 (F.D.C.) 24 examinations } \\
\hline First tertile & 0.659 & $<0.0001$ \\
\hline Second tertile & 0.833 & $<0.0001$ \\
\hline Third tertile & 0.750 & $<0.0001$ \\
\hline Global score & 0.842 & $<0.0001$ \\
\hline \multicolumn{3}{|c|}{ Investigator $\mathbf{2}$ (J.M.) 23 examinations } \\
\hline First tertile & 0.875 & $<0.0001$ \\
\hline Second tertile & 0.756 & $<0.0001$ \\
\hline Third tertile & 0.821 & $<0.0001$ \\
\hline Global score & 0.745 & $<0.0001$ \\
\hline \multicolumn{3}{|c|}{ Investigator 3 (M.J.M.) 23 examinations } \\
\hline First tertile & 0.950 & $<0.0001$ \\
\hline Second tertile & 0.906 & $<0.0001$ \\
\hline Third tertile & 0.939 & $<0.0001$ \\
\hline Global score & 0.928 & $<0.0001$ \\
\hline
\end{tabular}

Table 4 Interobserver agreement between each investigator and the central reader for each tertile and the global score: intraclass correlation coefficients.

\begin{tabular}{|c|c|c|c|}
\hline & ICC & $95 \% \mathrm{Cl}$ & $P$ \\
\hline \multicolumn{4}{|c|}{ Investigator 1 (F.D.C.) 24 examinations } \\
\hline First tertile & 0.788 & $0.510-0.908$ & $<0.0001$ \\
\hline Second tertile & 0.888 & $0.742-0.952$ & $<0.0001$ \\
\hline Third tertile & 0.857 & $0.669-0.938$ & $<0.0001$ \\
\hline Global score & 0.906 & $0.783-0.959$ & $<0.0001$ \\
\hline \multicolumn{4}{|c|}{ Investigator 2 (J.M.) 23 examinations } \\
\hline First tertile & 0.871 & $0.697-0.945$ & $<0.0001$ \\
\hline Second tertile & 0.824 & $0.586-0.926$ & $<0.0001$ \\
\hline Third tertile & 0.880 & $0.718-0.949$ & $<0.0001$ \\
\hline Global score & 0.852 & $0.650-0.937$ & $<0.0001$ \\
\hline \multicolumn{4}{|c|}{ Investigator 3 (M.J.M.) 23 examinations } \\
\hline First tertile & 0.971 & $0.933-0.988$ & $<0.0001$ \\
\hline Second tertile & 0.943 & $0.866-0.976$ & $<0.0001$ \\
\hline Third tertile & 0.968 & $0.925-0.987$ & $<0.0001$ \\
\hline Global score & 0.960 & $0.905-0.983$ & $<0.0001$ \\
\hline
\end{tabular}

ICC, intraclass correlation coefficient; $\mathrm{Cl}$, confidence interval. 


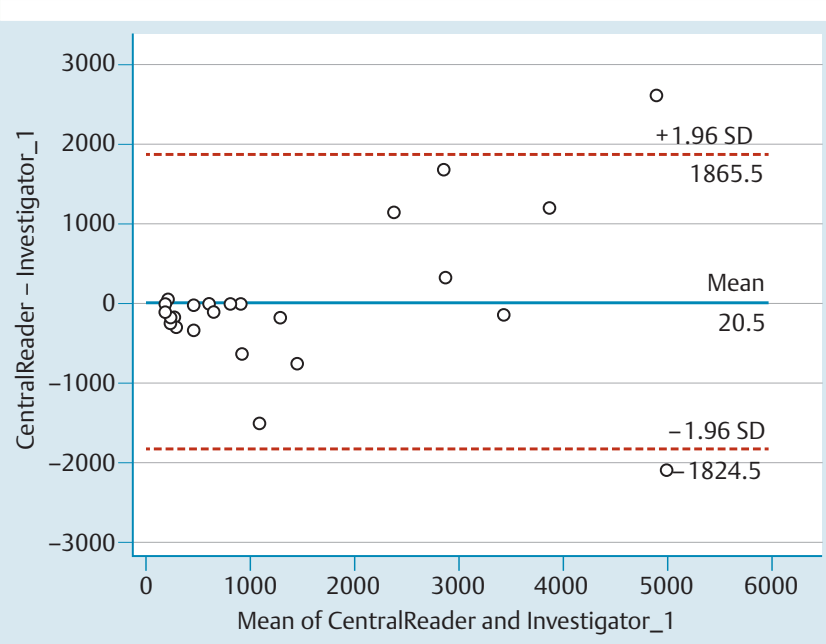

Fig. 1 Bland-Altman plot 1: interobserver agreement for global Lewis score between investigator 1 and the central reader.

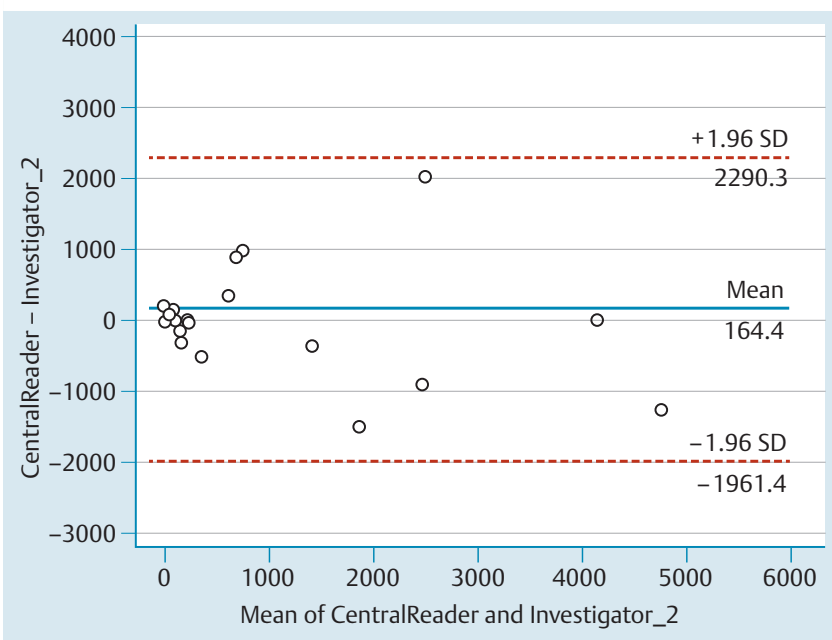

Fig. 2 Bland-Altman plot 2: interobserver agreement for global Lewis score between investigator 2 and the central reader.

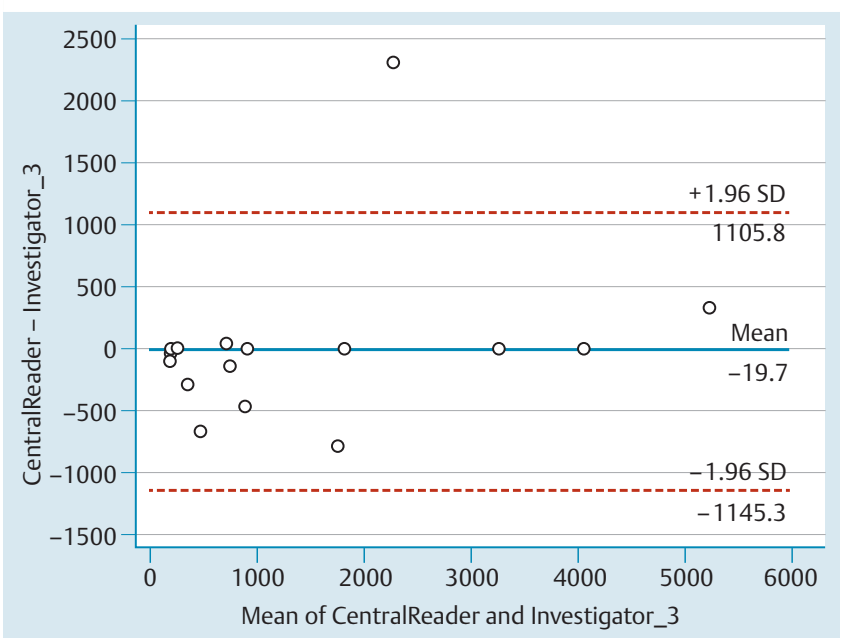

Fig. 3 Bland-Altman plot 3: interobserver agreement for global Lewis score between investigator 3 and the central reader.

\section{Discussion}

This study demonstrated that the Lewis score is a reproducible test to evaluate established Crohn's disease. There was strong interobserver agreement for the determination of the Lewis score, validating this score for the reporting of small-bowel inflammatory activity. Thus, the Lewis score might be used in clinical practice for diagnosing, staging, follow-up, and therapeutic assessment of patients with small-bowel Crohn's disease.

Globally, mean scores in each tertile and the global Lewis score were similar between the investigators and the central reader. There was a high or very high correlation for scoring results with almost perfect interobserver agreement. Interestingly, the analysis of Bland-Altman plots indicates that interobserver agreement may be lower for the highest scores, considering absolute numeric values, although it does not seem to influence the assessment of global disease severity according to the classification of the Lewis score, which stratifies small-bowel inflammatory activity into three grades $(<135$, normal or clinically insignificant mucosal inflammatory change; $\geq 135-<790$, mild disease; and $\geq 790$, moderate-to-severe disease). Indeed, overall, the differences between investigators, according to this classification, did not affect the global assessment of disease severity, which is clinically meaningful: to date, no study has evaluated whether there are different clinical or prognostic implications associated with scores of different magnitude above the cutoff of 790 , and therefore there is uncertainty over whether these differences may be clinically relevant.

The availability of an objective scoring system to assess inflammatory activity and disease extent enables the use of a common language and methodology for the interpretation and description of lesions consistent with Crohn's disease, and it may contribute to the establishment of cutoff levels indicative of the grade of severity with an adequate interobserver agreement. The adoption of reproducible scoring systems has been encouraged by recently published European Crohn's and Colitis Organisation guidelines [1]. The Lewis score $[7,10]$ is a cumulative scoring system based on the presence and distribution of villous edema, ulceration, and stenosis, which were the endoscopic findings with the highest interobserver agreement according to the original authors of the score $(\mathrm{K}=0.48, \mathrm{~K}=0.66$, and $\mathrm{K}=0.58$, respectively). Due to low interobserver agreement and/or perceived lack of clinical significance, other mucosal findings such as minor mucosal breaks, erythema, villous atrophy, or nodularity [10] were not included for the calculation of the score. The easy-to-use software application for the automatic calculation of the Lewis score is a clear advantage of the instrument compared with CECDAI, which currently has no software tool for its automatic calculation. Moreover, no threshold levels of inflammatory activity have been defined for CECDAI, although it has been suggested that CECDAI levels of 3.8 and 5.8 may correspond to the Lewis score thresholds of 135 and 790 , respectively [11].

A wide range of different clinical scenarios require the objective assessment of small-bowel inflammatory activity in clinical practice. In patients with suspected Crohn's disease after nondiagnostic ileocolonoscopy, it is possible to confidently exclude the diagnosis if no significant small-bowel mucosal changes are identified by the capsule, because the capsule has high sensitivity even for subtle mucosal changes $[6,12]$. Conversely, SBCE may be a key diagnostic tool in this setting if it reveals small-bowel lesions consistent with Crohn's disease in a proper clinical context. In this study, the gold standard for the diagnosis of Crohn's dis- 
ease did not rely solely on SBCE findings, but resulted from a combination of all available follow-up data, as described in the Methods section. Indeed, the diagnosis of Crohn's disease is confirmed histologically in only a minority of patients in routine clinical practice [13], either due to the inaccessibility of lesions or because biopsies are also often unspecific. Thus, usually the diagnosis is based on a conjunction of clinical, biochemical, endoscopic, and imaging elements. In our center, we do not routinely perform flexible enteroscopy to obtain biopsies of small-bowel lesions that are consistent with Crohn's disease as detected by SBCE if the patient has a high pre-test probability of Crohn's disease. In a recent study from our center in patients with suspected Crohn's disease, the diagnosis was confirmed during follow-up in $82.6 \%$ of those with significant inflammatory activity on SBCE (Lewis score $\geq 135$ ), but in only $12.1 \%$ of those with a score lower than 135 [14].

However, although the Lewis score can be used to quantitatively describe the type, distribution, and severity of mucosal lesions, endoscopic findings are nonspecific [4], and scoring systems grade inflammatory activity independently of its etiology. Indeed, lesions resembling Crohn's disease on SBCE are indistinguishable from NSAID-induced enteropathy or other causes of small-bowel mucosal damage such as infections, Behçet disease, ischemia, or vasculitis [1]. To date, no study has addressed whether the interobserver variability could be influenced by the type of indication for SBCE, namely whether the inflammatory activity would be graded differently in patients with suspected Crohn's disease or an alternative diagnosis compared with those with a previously known established diagnosis of Crohn's disease. Although this seems unlikely when a standard, objective, and validated methodology for the assessment of inflammatory activity is followed, this is an interesting topic of investigation that could merit further investigation.

The role of SBCE in patients with previously established Crohn's disease is currently evolving. It may be used to evaluate disease extent and activity in the small bowel, as proximal and/or extended inflammatory activity has been shown to be associated with poor prognosis and adverse outcomes $[15,16]$, and it may have an impact on therapeutic management towards an earlier introduction of immunomodulators and/or biologic therapy [17-19]. In a recently published retrospective study from our center, treatment with thiopurines and/or biologics was started more often in patients with proximal small-bowel lesions [13/33 (39\%) vs. $1 / 17$ (6\%); $P=0.011$, relative risk 6.5$]$, particularly when severe as determined by the Lewis score $(6 \%, 36 \%$, and $45 \%$ of patients with nonsignificant, mild, and moderate-to-severe inflammation, respectively) [18]. SBCE may also be considered in the postoperative setting $[20,21]$, or to investigate patients with atypical symptoms, unexplained anemia, or obscure gastrointestinal bleeding [1]. SBCE has also been evaluated as a noninvasive diagnostic tool for the assessment of mucosal healing after therapy, an important end point of treatment efficacy, which has been associated with sustained clinical remission and improved outcomes $[1,22,23]$. For all these indications, the objective quantification of small-bowel inflammatory activity by using a validated scoring methodology seems pivotal.

In this study, a strong correlation was demonstrated between the investigators and the central reader, both for scores obtained for each tertile as well as for the global Lewis score. Although the study was retrospective, it was based on prospectively collected database. A strong interobserver agreement was demonstrated for the Lewis score, validating this score for the reporting of small-bowel inflammation in a practical clinical setting. Previous studies have evaluated the correlation of endoscopic scoring systems with clinical objective measures, such as the Crohn's disease activity index or the inflammatory bowel disease questionnaire. Although this could be a topic for future investigations, several previous trials have failed to demonstrate an association between endoscopic scores and clinical scores in Crohn's disease [9,24, 25]. This may be due to the fact that clinical activity is objectively difficult to assess, and also because symptoms such as abdominal pain or diarrhea that may resemble Crohn's disease flare-ups may be multifactorial and unrelated to the existence of prominent gastrointestinal inflammatory lesions [9,24,25].

To summarize, the Lewis score has been shown to be a practical and reproducible instrument that might be used in the diagnosis, staging, follow-up, and therapeutic assessment of patients with isolated small-bowel Crohn's disease.

Competing interests: Dr. Rosa is a consultant for Given Imaging.

\section{Acknowledgments \\ $\nabla$}

The authors gratefully acknowledge the contribution of Firmino Machado, MD, for the critical statistical revision of the manuscript.

\section{References}

1 Annese V, Daperno M, Rutter MD et al. European evidence based consensus for endoscopy in inflammatory bowel disease. J Crohns Colitis 2013; 7: 982 - 1018

2 Dionisio PM, Gurudu SR, Leighton JA et al. Capsule endoscopy has a significantly higher diagnostic yield in patients with suspected and established small-bowel Crohn's disease: a meta-analysis. Am J Gastroenterol 2010; 105: $1240-1248$

3 Jensen MD, Nathan T, Rafaelsen SR et al. Diagnostic accuracy of capsule endoscopy for small bowel Crohn's disease is superior to that of MR enterography or CT enterography. Clin Gastroenterol Hepatol 2011; 9: $124-129$

4 Solem CA, Loftus EVJr, Fletcher JG et al. Small-bowel imaging in Crohn's disease: a prospective, blinded, 4-way comparison trial. Gastrointest Endosc 2008; 68: 255-266

5 Mow WS, Lo SK, Targan SR et al. Initial experience with wireless capsule enteroscopy in the diagnosis and management of inflammatory bowel disease. Clin Gastroenterol Hepatol 2004; 2: 31 - 40

6 Tukey M, Pleskow D, Legnani P et al. The utility of capsule endoscopy in patients with suspected Crohn's disease. Am J Gastroenterol 2009; 104: $2734-2739$

7 Gralnek IM, Defranchis R, Seidman E et al. Development of a capsule endoscopy scoring index for small bowel mucosal inflammatory change. Aliment Pharmacol Ther 2008; 27: 146-154

8 Gal E, Geller A, Fraser G et al. Assessment and validation of the new capsule endoscopy Crohn's disease activity index (CECDAI). Dig Dis Sci 2008; 53: 1933 - 1937

9 Niv Y, Ilani S, Levi Z et al. Validation of the Capsule Endoscopy Crohn's Disease Activity Index (CECDAI or Niv score): a multicenter prospective study. Endoscopy 2012; 44: $21-26$

10 Lewis BS. Expanding role of capsule endoscopy in inflammatory bowel disease. World J Gastroenterol 2008; 14: 4137-4141

11 Koulaouzidis A, Douglas S, Plevris JN. Lewis score correlates more closely with fecal calprotectin than Capsule Endoscopy Crohn's Disease Activity Index. Dig Dis Sci 2012; 57: 987 -993

12 Figueiredo $P$, Almeida $N$, Lopes $S$ et al. Small-bowel capsule endoscopy in patients with suspected Crohn's disease-diagnostic value and complications. Diagn Ther Endosc 2010: DOI: DOI 10.1155/2010/101284

13 Van Assche G, Dignass A, Panes J et al. The second European evidencebased consensus on the diagnosis and management of Crohn's disease: definitions and diagnosis. J Crohns Colitis 2010; 4: 7-27

14 Rosa B, Moreira MJ, Rebelo A et al. Lewis Score: a useful clinical tool for patients with suspected Crohn's Disease submitted to capsule endoscopy. J Crohns Colitis 2012; 6: 692-697 
15 Flamant $M$, Trang $C$, Maillard $O$ et al. The prevalence and outcome of jejunal lesions visualized by small bowel capsule endoscopy in Crohn's disease. Inflamm Bowel Dis 2013; 19: 1390 - 1396

16 Park SK, Yang SK, Park SH et al. Long-term prognosis of the jejunal involvement of Crohns disease. J Clin Gastroenterol 2013; 47: 400-408

17 Kalla R, McAlindon ME, Drew $K$ et al. Clinical utility of capsule endoscopy in patients with Crohn's disease and inflammatory bowel disease unclassified. Eur J Gastroenterol Hepatol 2013; 25: 706 -713

18 Cotter J, Dias de Castro F, Moreira MJ et al. Tailoring Crohn's disease treatment: the impact of small bowel capsule endoscopy. J Crohns Colitis In press 2014: DOI 10.1016/j.crohns.2014.02.018

19 Long MD, Barnes E, Isaacs K et al. Impact of capsule endoscopy on management of inflammatory bowel disease: a single tertiary care center experience. Inflamm Bowel Dis 2011; 17: 1855-1862

20 Pons Beltran V, Nos P, Bastida $G$ et al. Evaluation of postsurgical recurrence in Crohn's disease: a new indication for capsule endoscopy? Gastrointest Endosc 2007; 66: 533-540
21 Bourreille A, Jarry M, D'Halluin PN et al. Wireless capsule endoscopy versus ileocolonoscopy for the diagnosis of postoperative recurrence of Crohn's disease: a prospective study. Gut 2006; 55: 978-983

22 Efthymiou A, Viazis N, Mantzaris G et al. Does clinical response correlate with mucosal healing in patients with Crohn's disease of the small bowel? A prospective, case-series study using wireless capsule endoscopy Inflamm Bowel Dis 2008; 14: $1542-1547$

23 Swaminath A, Legnani P, Kornbluth A. Video capsule endoscopy in inflammatory bowel disease: past, present, and future redux. Inflamm Bowel Dis 2010; 16: $1254-1262$

24 Denis MA, Reenaers C, Fontaine F et al. Assessment of endoscopic activity index and biological inflammatory markers in clinically active Crohn's disease with normal C-reactive protein serum level. Inflamm Bowel Dis 2007; 13: 1100-1105

25 Minderhoud IM, Oldenburg B, Wismeijer JA et al. IBS-like symptoms in patients with inflammatory bowel disease in remission; relationships with quality of life and coping behavior. Dig Dis Sci 2004; 49: 469-474 